\title{
Rayleigh-Taylor instability and mushroom-pattern formation in a two-component Bose-Einstein condensate
}

\author{
Kazuki Sasaki, ${ }^{1}$ Naoya Suzuki, ${ }^{1}$ Daisuke Akamatsu, ${ }^{2}$ and Hiroki Saito ${ }^{1}$ \\ ${ }^{1}$ Department of Applied Physics and Chemistry, University of Electro-Communications, Tokyo 182-8585, Japan \\ ${ }^{2}$ National Metrology Institute of Japan (NMIJ), National Institute of Advanced Industrial Science and Technology (AIST), \\ Tsukuba 305-8563, Japan
}

(Received 8 October 2009; published 4 December 2009)

\begin{abstract}
The Rayleigh-Taylor instability at the interface in an immiscible two-component Bose-Einstein condensate is investigated using the mean field and Bogoliubov theories. Rayleigh-Taylor fingers are found to grow from the interface and mushroom patterns are formed. Quantized vortex rings and vortex lines are then generated around the mushrooms. The Rayleigh-Taylor instability and mushroom-pattern formation can be observed in a trapped system.
\end{abstract}

DOI: 10.1103/PhysRevA.80.063611

PACS number(s): 03.75.Mn, 03.75.Kk, 47.20.Ma

\section{INTRODUCTION}

When a layer of a lighter fluid lies under that of a heavier fluid, the translation symmetry on the interface is spontaneously broken and the interface is modulated due to the Rayleigh-Taylor instability (RTI) [1-4]. Waves on the interface then grow into complicated patterns with mushroom shapes $[3,5]$. The RTI plays crucial roles in a variety of nonequilibrium phenomena, ranging from convection of water in a kettle to supernova explosions [6].

In the present paper, we investigate the RTI and ensuing dynamics in a phase-separated two-component Bose-Einstein condensate (BEC). Recently, there has been a growing interest in the surface and interface properties of BECs. For instance, the Kelvin-Helmholtz instability $[4,7,8]$, which occurs at the interface between two fluids with a relative velocity, has been observed in a ${ }^{3} \mathrm{He}$ superfluid system $[9,10]$. The Kelvin-Helmholtz instability is also predicted in a two-component BEC of atomic gases [11]. When a magnetic field is applied to a magnetic fluid (a colloidal suspension of fine magnetic particles), the surface is deformed by the Rosensweig instability [12] and grows into a pattern of crests. Such a surface phenomenon can be theoretically shown to occur also at the interface in a two-component BEC with a dipole-dipole interaction [13]. Analytical expressions of the interface tension in a two-component BEC have been derived in [14-16]. The RTI at the surface of expanding two dimensional BECs of excitons and atomic gases is predicted in [17]. The RTI in a binary BEC using the Feshbach resonance is proposed in [18].

The present paper reveals that the RTI emerges at the interface between two immiscible BECs that are pushed toward each other by a magnetic field gradient. We first consider an ideal flat interface, and numerically show that the interface becomes deformed by the RTI to grow into the well-known mushroom pattern. The significant difference between this phenomenon and that in classical fluids is that the vortices under the caps of the mushrooms are quantized. Thus, in three dimensions (3D), quantized vortex rings are generated around the mushrooms. Bogoliubov analysis shows that the excitation spectrum of the interface modes closely resembles that for classical fluids. We also propose a realistic BEC system in a harmonic trap and show that the RTI can be observed experimentally.

This paper is organized as follows. Section II A numerically formulates the problem and demonstrates the RTI and ensuing dynamics for an ideal system. Section II B gives the Bogoliubov spectrum of the interface modes. Section III analyzes a trapped system. Section IV provides conclusions to the study.

\section{RAYLEIGH-TAYLOR INSTABILITY AT AN IDEAL INTERFACE}

\section{A. Mean-field dynamics}

The system considered here is a zero-temperature twocomponent BEC described by the Gross-Pitaevskii (GP) equations,

$$
\begin{aligned}
& i \hbar \frac{\partial \psi_{1}}{\partial t}=\left[-\frac{\hbar^{2}}{2 m_{1}} \nabla^{2}+V_{1}(\mathbf{r})+g_{11}\left|\psi_{1}\right|^{2}+g_{12}\left|\psi_{2}\right|^{2}\right] \psi_{1}, \\
& i \hbar \frac{\partial \psi_{2}}{\partial t}=\left[-\frac{\hbar^{2}}{2 m_{2}} \nabla^{2}+V_{2}(\mathbf{r})+g_{22}\left|\psi_{2}\right|^{2}+g_{12}\left|\psi_{1}\right|^{2}\right] \psi_{2},
\end{aligned}
$$

where $\psi_{j}, m_{j}$, and $V_{j}$ are the macroscopic wave function, atomic mass, and external potential, respectively, for the $j$ th $(j=1,2)$ component. The interaction parameters $g_{j j^{\prime}}$ are given by

$$
g_{j j^{\prime}}=\frac{2 \pi \hbar^{2} a_{j j^{\prime}}}{m_{j j^{\prime}}},
$$

where $a_{j j^{\prime}}$ and $m_{j j^{\prime}}$ are the $s$-wave scattering length and reduced mass, respectively, between components $j$ and $j^{\prime}$. We assume that the interaction parameters satisfy the phaseseparation condition,

$$
g_{11} g_{22}<g_{12}^{2}
$$

For concreteness, we employ the hyperfine states $\left|F, m_{F}\right\rangle=|1,1\rangle$ and $|1,-1\rangle$ of $\mathrm{a}{ }^{87} \mathrm{Rb}$ atom for components 1 


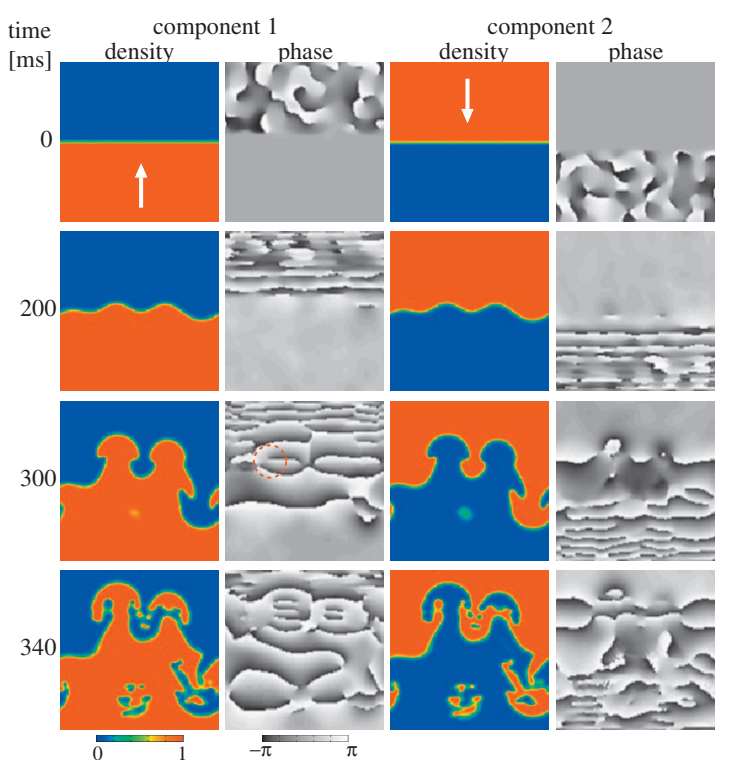

FIG. 1. (Color) Density and phase profiles on the $x-z$ plane. The initial state is a quasistationary state with a small random seed. The white arrows show the directions of the Stern-Gerlach force produced by the field gradient of $B^{\prime}=20 \mathrm{mG} / \mathrm{cm}$. The red circle indicates the location of a topological defect under a mushroom cap. The density is normalized by $4 \times 10^{14} \mathrm{~cm}^{-3}$. The field of view is $100 \times 100 \mu \mathrm{m}$.

and 2, respectively. According to the scattering lengths reported in [19], we have $a_{11}=a_{22}=100.4 a_{\mathrm{B}}$ and $a_{12}=101.3 a_{\mathrm{B}}$ with $a_{\mathrm{B}}$ being the Bohr radius, and then the condition for the phase separation in Eq. (3) is satisfied. The spin-exchange dynamics $|1,1\rangle,|1,-1\rangle \rightarrow|1,0\rangle,|1,0\rangle$ can be suppressed by, e.g., the microwave-induced quadratic Zeeman effect [20], which can lift the energy of the $|1,0\rangle$ state. When the hyperfine spins are parallel to the magnetic field $\boldsymbol{B}$, the magnetic field gradient exerts forces $\pm \mu_{B} \nabla|\boldsymbol{B}| / 2$ on the two components in opposite directions, where $\mu_{\mathrm{B}}$ is the Bohr magneton.

In order to clearly demonstrate the RTI, we first consider a $3 \mathrm{D}$ system without a trapping potential. We prepare a quasi-stationary state with a field gradient $B^{\prime} \equiv d B / d z>0$, which is uniform in the $x-y$ direction. Components 1 and 2 are located in the $z<0$ and $z>0$ regions, respectively, and their flat interface is located at the $z=0$ plane. The SternGerlach force pushes the two components toward each other. We add small random seeds to the initial state, which numerically breaks the translation symmetry in the $x$-y direction and triggers the RTI. We assume periodic boundary conditions in the $x$ and $y$ directions.

Figure 1 shows the time evolution of the density and phase profiles on a plane perpendicular to the initial flat interface. The two components are pushed toward each other (arrows in Fig. 1), and the interface starts to modulate due to the RTI (second row of Fig. 1). Subsequently, the amplitude of the wave on the interface grows to form the mushroom shapes (third row of Fig. 1). We can see that there are quantized vortices under the caps of the mushrooms (red circle in Fig. 1). After that, the vortices enter into the mushroom patterns, giving rise to complicated dynamics (fourth row of Fig. 1).

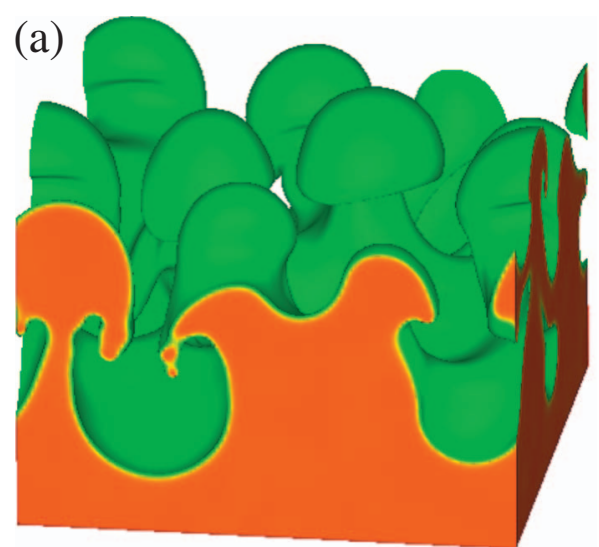

(b)

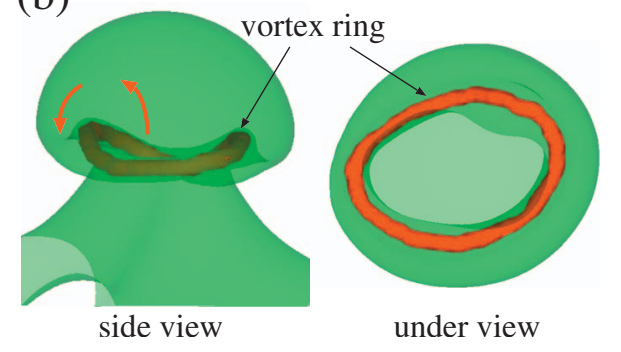

FIG. 2. (Color) (a) Isodensity surface of component 1 at $t=310 \mathrm{~ms}$. The condition is the same as that in Fig. 1. (b) One of the mushroom shapes in (a). The red rings show the location of the topological defects. The red arrows indicate the directions of the atomic flow.

Figure 2(a) shows the isodensity surface of component 1 at $t=310 \mathrm{~ms}$. We can see that Rayleigh-Taylor fingers and mushroom patterns flourish at the interface. One of the mushroom shapes is magnified in Fig. 2(b), in which the topological defect under the cap of the mushroom is indicated by the red ring. The vortex rings are generated by the upward flow of the atoms around the center and downward flow at the periphery of the cap of the mushroom shape.

\section{B. Bogoliubov analysis}

Before studying the Bogoliubov spectrum, we recall the dispersion relation for the RTI in classical fluids. Let us consider a situation, in which inviscid incompressible fluids produce a flat interface perpendicular to the direction of gravity, where the densities of the lower and upper fluids are $\rho_{1}$ and $\rho_{2}$ with $\rho_{1}<\rho_{2}$. From the linear analysis, the interface mode has a dispersion relation of [4]

$$
\omega=\left[\frac{\left(\rho_{1}-\rho_{2}\right) g k+\sigma k^{3}}{\rho_{1}+\rho_{2}}\right]^{1 / 2},
$$

where $g$ is the gravitational constant and $\sigma$ is an interfacetension coefficient. If gravity is absent, $\omega$ is real for all $k$ and proportional to $k^{3 / 2}$. In the presence of the gravitational force, there is always a range of $k$ in which $\omega$ is imaginary, and hence the interface is dynamically unstable. The range of instability is given by 


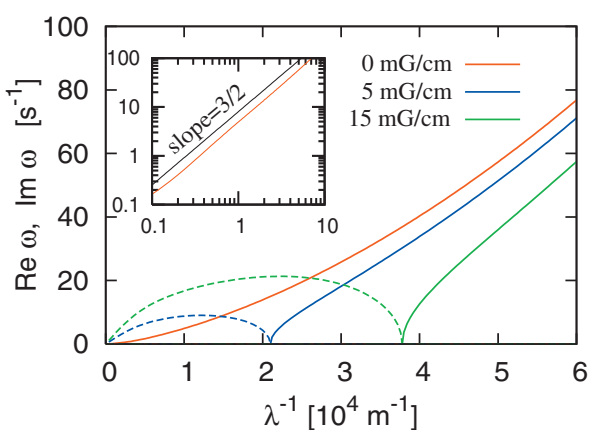

FIG. 3. (Color) Real part (solid lines) and imaginary part (dashed lines) of the Bogoliubov spectrum of an interface mode for a flat interface. The field gradient perpendicular to the interface is $B^{\prime}=0$ (red), 5 (blue), and $15 \mathrm{mG} / \mathrm{cm}$ (green). The red line in the inset shows a logarithmic plot for $B^{\prime}=0$. For comparison, a black line with a slope of $3 / 2\left(\omega \propto \lambda^{-3 / 2}\right)$ is shown in the inset.

$$
0<k<\sqrt{\frac{\left(\rho_{2}-\rho_{1}\right) g}{\sigma}} \equiv 2 \pi \lambda_{\mathrm{c}}^{-1},
$$

and the most unstable wave number $k_{0}$ is

$$
k_{0}=\sqrt{\frac{\left(\rho_{2}-\rho_{1}\right) g}{3 \sigma}} .
$$

We perform the Bogoliubov analysis by decomposing the wave function as

$$
\psi_{j}(\boldsymbol{r}, t)=\left[f_{j}(z)+\phi_{j}(\boldsymbol{r})\right] e^{-i \mu_{j} t} \quad(j=1,2),
$$

where $f_{j}(z)$ is a quasistationary state with a flat interface around the $z=0$ plane and $\mu_{j}$ is the chemical potential. For the case of Fig. $1, f_{1}(z)$ and $f_{2}(z)$ are symmetric with respect to the interface on the $x-y$ plane and $\mu_{1}=\mu_{2} \equiv \mu$ because $g_{11}=g_{22}$. The small deviation $\phi_{j}(\boldsymbol{r})$ from the ground state is written as

$$
\phi_{j}(\boldsymbol{r})=u_{j, k}(z) e^{i(k x-\omega t)}+v_{j, k}^{*}(z) e^{-i(k x-\omega t)},
$$

where the wave vector is assumed to be in the $x$ direction without loss of generality. The mode functions $u_{j, k}(z)$ and $v_{j, k}(z)$ satisfy the Bogoliubov-de Gennes equations,

$$
\begin{aligned}
& {\left[-\frac{\hbar^{2}}{2 m}\left(\frac{\partial^{2}}{\partial z^{2}}+k^{2}+V_{j}\right)-\mu+2 g_{j j} f_{j}^{2}+g_{j j^{\prime}} f_{j^{\prime}}^{2}\right] u_{j, k}+g_{j j} f_{j}^{2} v_{j, k}} \\
& +g_{j j^{\prime}} f_{j} f_{j^{\prime}}\left(u_{j^{\prime}, k}+v_{j^{\prime}, k}\right)=\hbar \omega u_{j, k}, \\
& {\left[-\frac{\hbar^{2}}{2 m}\left(\frac{\partial^{2}}{\partial z^{2}}+k^{2}+V_{j}\right)-\mu+2 g_{j j} f_{j}^{2}+g_{j j^{\prime}} f_{j^{\prime}}^{2}\right] v_{j, k}+g_{j j} f_{j}^{2} u_{j, k}} \\
& +g_{j j^{\prime}} f_{j} f_{j^{\prime}}\left(u_{j^{\prime}, k}+v_{j^{\prime}, k}\right)=-\hbar \omega v_{j, k},
\end{aligned}
$$

where $\left(j, j^{\prime}\right)=(1,2)$ and $(2,1), m$ is the mass of ${ }^{87} \mathrm{Rb}$, $V_{1}=-\mu_{B} B^{\prime} z / 2, V_{2}=\mu_{B} B^{\prime} z / 2$, and $f_{j}$ is assumed to be real.

Figure 3 shows the Bogoliubov spectrum obtained by numerically diagonalizing Eq. (9). In Fig. 3, we plot only the lowest mode, which corresponds to the mode localized near the interface. The second lowest mode has much larger energy. When the field gradient $B^{\prime}$ is zero, the excitation energy is real for all wavelengths $\lambda$ (red line in Fig. 3). The loga-

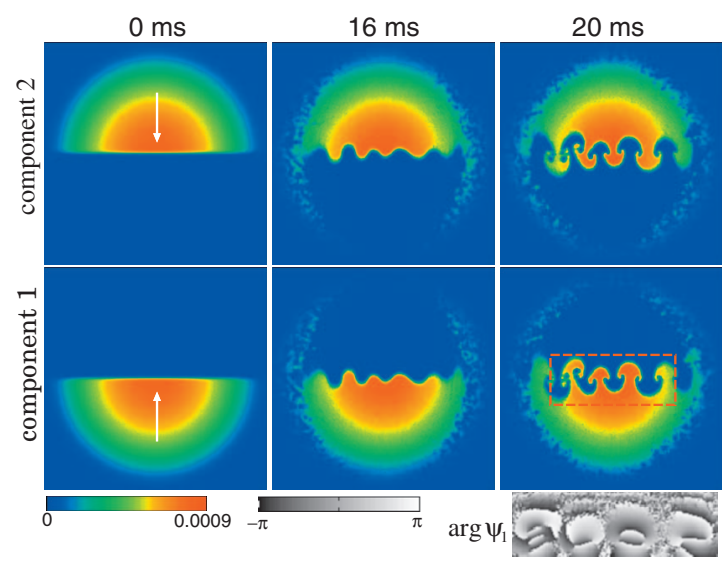

FIG. 4. (Color) Column density $\int\left|\psi_{j}\right|^{2} d y$ of the two-component BEC in a tight pancake-shaped trap with $\omega_{x z}=2 \pi \times 100 \mathrm{~Hz}$ and $\omega_{y}=2 \pi \times 5 \mathrm{kHz}$. The number of atoms is $N=8.1 \times 10^{6}$ with an equal population in each component. The initial state is the ground state for $B^{\prime}=0$ plus a small random seed. At $t=0$, the field gradient is changed to $B^{\prime}=1.3 \mathrm{G} / \mathrm{cm}$. The white arrows show the directions of the Stern-Gerlach force for the two components. The unit of the density is $\left(m \omega_{x z} / \hbar\right)^{3 / 2}$. The field of view is $64 \times 64 \mu \mathrm{m}$. The phase profile in the red square is shown in the grayscale image.

rithmic plot in the inset of Fig. 3 indicates that $\omega$ is proportional to $k^{3 / 2}$, which is in agreement with Eq. (4) with $g=0$. In the presence of the field gradient $B^{\prime}$, the Bogoliubov spectrum becomes imaginary for $\lambda^{-1}$ smaller than a critical value $\lambda_{\mathrm{c}}^{-1}$, which is $\lambda_{\mathrm{c}}^{-1} \simeq 2.1 \times 10^{4} \mathrm{~m}^{-1}$ for $B^{\prime}=5 \mathrm{mG} / \mathrm{cm}$ and $\lambda_{\mathrm{c}}^{-1} \simeq 3.8 \times 10^{4} \mathrm{~m}^{-1}$ for $B^{\prime}=15 \mathrm{mG} / \mathrm{cm}$. The longwavelength modes are always unstable for $B^{\prime} \neq 0$, as in the RTI in classical fluids.

The analytic expression of the interface tension in a phase-separated two-component BEC has been derived in [14-16]. For $a_{12} / a-1 \ll 1$, where $a \equiv a_{11}=a_{22}$, the interface tension $\sigma$ has the form,

$$
\sigma=\frac{\hbar^{2} n^{3 / 2}}{m} \sqrt{2 \pi\left(a_{12}-a\right)},
$$

where $n$ is the atom density. Using the characteristic density $4 \times 10^{14} \mathrm{~cm}^{-3}$ for $n$ and substituting Eq. (10) into Eq. (5), in which $\left(\rho_{2}-\rho_{1}\right) g$ is replaced by $n \mu_{\mathrm{B}} B^{\prime}$, we obtain $\lambda_{\mathrm{c}}^{-1} \simeq 2.1$ $\times 10^{4} \mathrm{~m}^{-1}$ for $B^{\prime}=5 \mathrm{mG} / \mathrm{cm}$ and $\lambda_{\mathrm{c}}^{-1} \simeq 3.6 \times 10^{4} \mathrm{~m}^{-1}$ for $B^{\prime}=15 \mathrm{mG} / \mathrm{cm}$. These values of $\lambda_{\mathrm{c}}^{-1}$ are in good agreement with those in Fig. 3. Using Eqs. (6) and (10), the most unstable wavelength is estimated to be $\simeq 40 \mu \mathrm{m}$ for $B^{\prime}$ $=20 \mathrm{mG} / \mathrm{cm}$, which is in qualitative agreement with the wavelength of the interface modulation in Fig. 1.

\section{DYNAMICS IN A HARMONIC TRAP}

We next consider a system confined in an axisymmetric harmonic potential $V_{\text {trap }}=m\left[\omega_{x z}^{2}\left(x^{2}+z^{2}\right)+\omega_{y}^{2} y^{2}\right] / 2$. The radial and axial trap frequencies are $\omega_{x z}=2 \pi \times 100 \mathrm{~Hz}$ and $\omega_{z}$ $=2 \pi \times 5 \mathrm{kHz}$, and the potential has a tight pancake shape. The initial state is the ground state of the GP equation for $B^{\prime}=0$, in which the interface is parallel to the $x$ axis and components 1 and 2 (the hyperfine states $|1,1\rangle$ and $|1,-1\rangle$ of 
${ }^{87} \mathrm{Rb}$ ) are localized in the $z<0$ and $z>0$ regions, respectively. A small random noise is added to the initial state to trigger the dynamical instability. At $t=0$, the field gradient $B^{\prime}=1.3 \mathrm{G} / \mathrm{cm}$ is applied in the $z$ direction.

Figure 4 shows the time evolution of the column density $\int\left|\psi_{j}\right|^{2} d y$ of each component, obtained by 3D simulation of the GP equation. At $t \simeq 80 \mathrm{~ms}$, the interface starts to modulate with a wavelength $\simeq 8-10 \mu \mathrm{m}$ due to the RTI. This wavelength is in qualitative agreement with $\simeq 8.7 \mu \mathrm{m}$ estimated using Eqs. (6) and (10) with the peak density $n$ $\simeq 3.2 \times 10^{15} \mathrm{~cm}^{-3}$. The modulation on the interface then develops into the mushroom patterns at $t \simeq 100 \mathrm{~ms}$. After that, the system evolves in a complicated manner and eventually components 1 and 2 are interchanged, localizing in the $z$ $>0$ and $z<0$ regions, respectively.

In Fig. 4, one can see that both components invade around to the back of each other and the periphery of the condensate is disturbed. This is because the repulsive interaction between the two components is weak in the low-density periphery region, and the two components pass through each other. For a spherical trap, the pass-through phenomenon is more severe and a much larger number of atoms $\left(>10^{9}\right)$ is needed to clearly realize the RTI. If we use a square-well potential produced by, e.g., a flat-top beam [21], the atomic density becomes more uniform on the interface, which suppresses the pass-through, realizing an ideal RTI as discussed in Sec. II. Increasing the intercomponent repulsion using the Fesh- bach resonance $[19,22,23]$ can also suppress the pass through.

\section{CONCLUSIONS}

We have shown that the RTI and mushroom-pattern formation occur in a two-component phase-separated BEC, as in classical fluids. The significant difference between the quantum RTI and the classical RTI is that quantized vortex lines and vortex rings are formed under the caps of the mushrooms. The Bogoliubov analysis showed that the excitation spectra of the interface modes are very similar to those of classical fluids. We proposed a possible experiment to observe the phenomena in a realistic trapped system.

Various phenomena related to fluid instabilities may be reproduced in BECs with renewed interest. For example, splashing of drops and crown formation [24] is considered to be related to instabilities which include the RTI. Droplet formation by the Plateau-Rayleigh instability [25] in BECs is also an interesting future problem.

\section{ACKNOWLEDGMENTS}

We thank S. Tojo for valuable comments. This work was supported by the Ministry of Education, Culture, Sports, Science and Technology of Japan (Grants-in-Aid for Scientific Research, Grants No. 17071005 and No. 20540388).
[1] Lord Rayleigh, Proc. London Math. Soc. s1-14, 170 (1882).

[2] G. Taylor, Proc. R. Soc. London, Ser. A 201, 192 (1950).

[3] D. J. Lewis, Proc. R. Soc. London Ser. A 202, 81 (1950).

[4] For a textbook, S. Chandrasekhar, Hydrodynamic and Hydromagnetic Stability (Clarendon Press, Oxford, 1961), Chap. 10.

[5] B. J. Daly, Phys. Fluids 10, 297 (1967).

[6] A. Burrows, Nature (London) 403, 727 (2000).

[7] H. von Helmholtz, Philos. Mag. 36, 337 (1868).

[8] Lord Kelvin, Philos. Mag. 42, 362 (1871).

[9] R. Blaauwgeers, V. B. Eltsov, G. Eska, A. P. Finne, R. P. Haley, M. Krusius, J. J. Ruohio, L. Skrbek, and G. E. Volovik, Phys. Rev. Lett. 89, 155301 (2002).

[10] G. E. Volovik, Pis'ma Zh. Eksp. Teor. Fiz. 75, 491 (2002) [JETP Lett. 75, 418 (2002)].

[11] H. Takeuchi, N. Suzuki, K. Kasamatsu, H. Saito, and M. Tsubota, e-print arXiv:0909.2144.

[12] M. D. Cowley and R. E. Rosensweig, J. Fluid Mech. 30, 671 (1967).

[13] H. Saito, Y. Kawaguchi, and M. Ueda, Phys. Rev. Lett. 102, 230403 (2009).
[14] P. Ao and S. T. Chui, Phys. Rev. A 58, 4836 (1998).

[15] R. A. Barankov, Phys. Rev. A 66, 013612 (2002).

[16] B. Van Schaeybroeck, Phys. Rev. A 78, 023624 (2008).

[17] S. Chatterjee, e-print arXiv:cond-mat/0512016.

[18] S. Gautam and D. Angom, e-print arXiv:0908.4336.

[19] E. G. M. van Kempen, S. J. J. M. F. Kokkelmans, D. J. Heinzen, and B. J. Verhaar, Phys. Rev. Lett. 88, 093201 (2002).

[20] S. R. Leslie, J. Guzman, M. Vengalattore, J. D. Sau, M. L. Cohen, and D. M. Stamper-Kurn, Phys. Rev. A 79, 043631 (2009).

[21] B. Hao, J. Burch, and J. Leger, Appl. Opt. 47, 2931 (2008).

[22] M. Erhard, H. Schmaljohann, J. Kronjäger, K. Bongs, and K. Sengstock, Phys. Rev. A 69, 032705 (2004).

[23] G. Thalhammer, G. Barontini, L. De Sarlo, J. Catani, F. Minardi, and M. Inguscio, Phys. Rev. Lett. 100, 210402 (2008).

[24] For review, see, A. L. Yarin, Annu. Rev. Fluid Mech. 38, 159 (2006).

[25] Lord Rayleigh, Proc. London Math. Soc. s1-10, 4 (1878); Proc. R. Soc. London, Ser. A 29, 71 (1879). 